\title{
Calcium phosphate nanoparticles: a future therapeutic platform for the treatment of osteomyelitis?
}

Keywords: antibiotics $=$ calcium phosphate $\|$ nanoparticles $\|$ osteomyelitis $\approx$ sustained drug delivery

"It is hard to be brave', said Piglet, sniffing slightly, 'when you're only a very small animal'. Rabbit, who had begun to write very busily, looked up and said: 'It is because you are a very small animal that you will be useful in the adventure before us'."

Alan Alexander Milne,

Winnie-the-Pooh and All, All, All

Osteomyelitis, one of the oldest documented diseases, the descriptions of which date back to times of Hippocrates [1], is an illness particularly prevalent among elderly, diabetics, children and indigenes of Third World countries. Although morbidity due to chronic bone infection has drastically dropped from the pre-penicillin era, down to approximately $3 \%$ in the last 20 years [2], it is still high on the global scale and the disease continues to be challenging to treat [3]. Furthermore, the number of hip and knee replacement procedures performed in the USA has doubled in the past decade, while the number of the reported cases of bone infection accompanying those has been increasing in proportion with the number of surgeries performed [4], signifying the anticipated increase in the incidence rate of this illness in the future.

The standard therapy for osteomyelitis has been based on the combination of repetitive intravenous or oral delivery of antibiotics over the period of a few weeks to a few months, depending of the severity of the infection, and surgical debridement of necrotic bone [5]. This clinical approach has had its obvious disadvantages, predominantly in terms of:

- Systemic administration of antibiotics and its side effects;

- Frequent inaccessibility of the infected zone to the blood flow and the resulting low concentration of the therapeutic around it, potentially inducing pathogenic resistance to the antibiotic therapy;
- Irretrievable bone loss;

- Cost-ineffectiveness.

For this reason, more advanced antibiotic delivery options have been actively sought since the early 1970s, when the first carriers for local delivery in the treatment of osteomyelitis, composed of poly(methyl methacrylate), were clinically applied [6]. Even though poly(methyl methacrylate) beads still present the gold standard for the local delivery of antibiotics to bone cavities, their downsides are numerous, including:

- Non-biodegradability;

- Proneness to biofilm formation;

- Need to be surgically removed;

- Tendency to exhibit burst release;

- Inconsistent release profiles, a corollary of the typically manual presurgical loading;

- Limited clinical data in support thereof [7].

The problem with calcium sulfates and collagen fleeces, two other commonly used antibiotic carriers in the clinic, comes from their high resorption rate and non-immunogenicity, respectively.

Nanosized calcium phosphates (CAPs), in contrast, present potentially the most natural choice for antibiotic delivery platforms in bone therapy. First of all, nano-CAP is the natural mineral component of bone. CAP crystals in bone, stiff but brittle, have the role of imparting sufficient compressive strength to intrinsically tough collagen fibers, thus yielding a composite material that is both strong and tough. Second, CAPs are bioactive, bioresorptive and osteoconductive, that is, conducive to bone growth. Third, they are one of the safest and most biocompatible nanomaterials assessed for toxicity

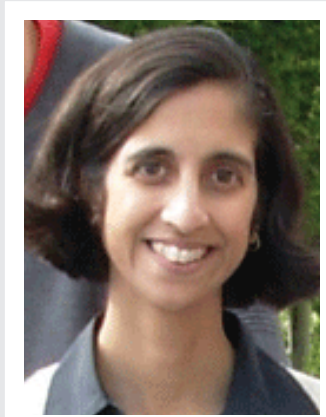

Tejal A Desai

Author for correspondence:

Therapeutic Micro \&

Nanotechnology Laboratory,

Department of Bioengineering \&

Therapeutic Sciences, University of

California, San Francisco, CA, USA

E-mail: tejal.desai@ucsf.edu

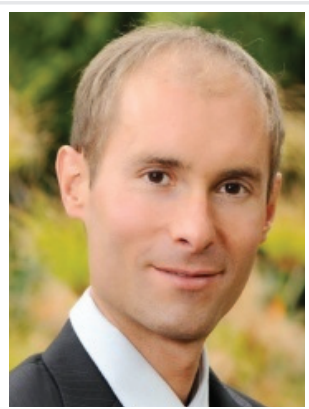

Vuk Uskoković

Therapeutic Micro \&

Nanotechnology Laboratory,

Department of Bioengineering \&

Therapeutic Sciences, University of

California, San Francisco, CA, USA

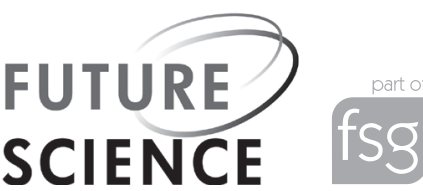


so far [8]. Fourth, they could be prepared in nanoform and in a variety of morphologies using simple and eco-friendly precipitation procedures [9]. Fifth, they are good adsorbents of biomolecules, which predisposes them to be used as the stationary phase in chromatographic columns [10] and is a prerequisite for achieving satisfying loading capacities. Finally, CAPs exist in a dozen or so different phases, whose solubility varies from highly soluble mono-CAPs to moderately soluble di-CAPs to sparsely soluble amorphous CAP and tri-CAPs to very sparsely soluble octa-CAP and hydroxyapatite, the CAP phase found in bone, dentin and enamel. The control of stochiometry of CAP thus makes it possible to tune its degradation rate and, therefore, the drug release kinetics. This effect has been employed to vary in vitro drug release time scale from hours to months [11].

Target areas in the body and types of the drugs delivered require specific release patterns for the optimal therapeutic effect to be achieved. For example, $\beta$-lactam antimicrobials are time-dependent, requiring prolonged presence in the target zone, while quinolones and aminoglycosides are concentration-dependent [12]. Then, vasculature of the target tissue affects the drug-clearance period, which is why different release profiles prove to be optimal for highly vascular trabecular bone and less vascular cortical bone. Easily achievable tunability of the release rate thus presents a highly desirable property of the drug carrier.

\section{"Target areas in the body and types of the drugs delivered require specific release patterns for the optimal therapeutic effect to be achieved."}

The capability of CAPs to augment the osteogenic response of osteoblasts, the bone-building cells, has been demonstrated on multiple occasions $[13,14] . \mathrm{Ca}^{2+}$ and $\mathrm{H}_{\mathrm{x}} \mathrm{PO}_{4}{ }^{\mathrm{x}-3}$ ions released upon the degradation of these compounds can also stimulate osteoblastic differentiation [15] and proliferation [16] and be used as ionic ingredients for the new bone formation. With systemic and repetitive administration of antibiotics and surgical debridement being two central problematic aspects of traditional therapies, an ideal delivery carrier would ensure not only local and sustained release of the drug, but also the induction of osteogenesis, so as to minimize, if not wholly eliminate, the surgical removal of affected bone. Patients with diabetic neuropathy are, for example, prone to develop osteomyelitis of the forefoot, which often leads to minor amputation [17]; however, with the development of osteogenic carriers that could revitalize the diseased bone, such clinical cases could be coped with in a manner less traumatic for the patient.

\section{"Although chemistry of calcium phosphates has been intensively studied for the last century, known facts about it still resemble the tip of an iceberg."}

A critical intrinsic downside of CAPs comes from the fact that intracrystalline impregnation with the drug via co-precipitation cannot be achieved, as in the case of polymers, which limits the loading mechanism to adsorption only and inevitably leads to burst release. Sustained release from CAPs is, however, accomplishable and seems to be contingent on the formation of compact blocks and encapsulation of the drug in the pores between the particles, so that the dissolution of the carrier proceeds along the block boundaries, while the bulk of the drug is being internally protected. This aggregation state, however, does not favor clinical injectability, a limitation that requires optimal additives to stabilize the surface drug layer and at the same time prevent particle coalescence.

These and similar deficiencies could be transcended by the usage of CAPs as the base for composite nanoparticles, the concept towards which the field of nanoscience unstoppably streams. Coating of CAP core with a layer of a poly $(\alpha$-hydroxy ester), such as poly(lactide-coglycolide), whose degradation rate itself could be tuned from a few years to a few weeks depending on the lactide-to-glycolide ratio, is an option to:

- Stabilize the surface-bound drug layer;

- Prevent the burst release;

- Possibly produce multiple-stage release profiles that may additionally boost the antimicrobial activity of the drug/carrier composite.

Coating chitosan/tri-CAP composites with poly ( $\varepsilon$-caprolactone) has thus mitigated the burst release and promoted zero-order kinetics for the release of vancomycin during the first 6 weeks [18]. Another important limitation of CAPs, difficult surface functionalization, a consequence of their ionic nature, which dictates that the surface layers would undergo rapid reorganization via dissolution/reprecipitation phenomena in ionic media, could be similarly overcome by coating 
the ceramic particles with chemically bondable polymeric layers. Through a series of chemical steps, polymeric coatings could further be conjugated with various targeting, solubilizing or therapeutic ligands [19]. Another interesting feature of the combinations of alkaline CAP phases, such as hydroxyapatite or octa-CAP, with acidic poly( $\alpha$-hydroxy esters), comes from their ability to compensate intense $\mathrm{pH}$ changes that follow the degradation of each of the two phases individually. This effect may explain why the addition of demineralized bone particles to poly(lactide-co-glycolide) was able to reduce inflammation, fibrous tissue encapsulation, and foreign body giant cell response [20].

CAPs, like most ceramics, possess a broad spectrum of possible stochiometries and structures, which could be, in theory, correlated with a similar, though largely unexplored, breadth of properties and applications. Although chemistry of CAPs has been intensively studied for the last century, known facts about it still resemble the tip of an iceberg. Not only is our knowledge of the mechanism of their formation, in biological milieus and simple reaction settings alike, still filled with vast gaps, but numerous other segments of their structure - property - function chain have yet to be elucidated. In the end, alongside all its qualities, CAP could be considered as the abovementioned Piglet in the kingdom of inorganic chemistry. What nature wished to teach us by choosing this pale, fragile, rough, crumbly and cheap compound as the ingredient of the structural basis of our physical beings is an open door to poetic insights that we will leave to the imagination of the reader and avid researchers of this remarkable material.

\section{Financial \& competing interests disclosure}

The authors acknowledge financial support from the National Institutes of Health award K99-DE021416. The authors have no other relevant affiliations or financial involvement with any organization or entity with a financial interest in or financial conflict with the subject matter or materials discussed in the manuscript apart from those disclosed.

No writing assistance was utilized in the production of this manuscript.

\section{References}

1 Lew DP, Waldvogel FA. Osteomyelitis. Lancet 364 (9431), 369-379 (2004).

2 Huckell CB, Kostuik JP. Pyogenic vertebral infection. In: Spinal Deformities: the Comprehensive Text. DeWald RL (Ed.). Thieme Medical Publishers, Inc., NY, USA, 222 (2003).

3 Ikpeme IA, Ngim NE, Ikpeme AA. Diagnosis and treatment of pyogenic bone infections. Afr. Health Sci. 10 (1), 82-88 (2010).

4 Del Pozo JL, Patel R. Infection associated with prosthetic joints. N. Eng. J. Med. 361, 787-794 (2009).

5 Hatzenbuehler J, Pulling TJ. Diagnosis and management of osteomyelitis. Am. Fam. Phys. 84 (9), 1027-1033 (2011).

6 Buchholz HW, Engelbrecht H. Depot effects of various antibiotics mixed with Palacos resins. Chirurg 41, 511-515 (1970).

7 Barth RE, Vogely HC, Hoepelman AI, Peters EJ. 'To bead or not to bead?' Treatment of osteomyelitis and prosthetic joint-associated infections with gentamicin bead chains. Int. J. Antimicrob. Agents. 38 (5), 371-375 (2011).

8 Singh N, Manshian B, Jenkins GJS et al. NanoGenotoxicology: the DNA damaging potential of engineered nanomaterials. Biomaterials 30, 3891-3914 (2009).
9 Uskoković V, Batarni SS, Schweicher J, King A, Desai TA. Effect of calcium phosphate particle shape and size on their antibacterial and osteogenic activity in the delivery of antibiotics in vitro. ACS Appl. Mat. Inter. 5(7), 2422-2431 (2013).

10 Saito M, Kurosawa Y, Okuyama T. Scanning electron microscopy-based approach to understand the mechanism underlying the adhesion of dengue viruses on ceramic hydroxyapatite columns. PLoS ONE 8(1), e53893 (2013).

11 Uskoković V, Desai TA. Phase composition control of calcium phosphate nanoparticles for tunable drug delivery kinetics and treatment of osteomyelitis. Part 1: preparation and drug release. J. Biomed. Mater. Res. A 101(5), 1416-1426 (2013).

12 Aarts HJM, Guerra B, Malorny B. Molecular methods for detection of antibiotic resistance. In: Antimicrobial Resistance in Bacteria of Animal Origin. Aarestrup FM (Ed.). American Society for Microbiology Press, DC, USA, 55 (2006).

13 Yuan H, Fernands H, Habibovic P et al. Osteoinductive ceramics as a synthetic alternative to autologous bone grafting. Proc. Natl Acad. Sci. USA 107, 13614-13619 (2010).

14 Rupani A, Hidalgo-Bastida LA, Rutten F, Dent A, Turner I, Cartmell S. Osteoblast activity on carbonated hydroxyapatite.
J. Biomed. Mater. Res. A 100(4), 1089-1096 (2012).

15 Liu YK, Lu QZ, Pei R et al. The effect of extracellular calcium and inorganic phosphate on the growth and osteogenic differentiation of mesenchymal stem cells in vitro: implication for bone tissue engineering. Biomed. Mater. 4(2), 025004 (2009).

16 Mailland M, Waelchli R, Ruat M, Boddeke HG, Seuwen K. Stimulation of cell proliferation by calcium and a calcimimetic compound. Endocrinology 138, 3601-3605 (1997).

17 Melamed EA, Peled E. Antibiotic impregnated cement spacer for salvage of diabetic osteomyelitis. Foot Ankle Int. 33(3), 213-219 (2012).

18 Fang T, Wen J, Zhou J, Shao Z, Dong J. Poly ( $\varepsilon$-caprolactone) coating delays vancomycin delivery from porous chitosan/ $\beta$-tricalcium phosphate composites. J. Biomed. Mater. Res. B 100 (7), 1803-1811 (2012).

19 Cheng J, Teply BA, Sherifi I et al. Formulation of functionalized PLGA-PEG nanoparticles for in vivo targeted drug delivery. Biomaterials 28, 869-876 (2007).

20 Yoon SJ, Kim SH, Ha HJ et al. Reduction of inflammatory reaction of poly(D,L-lactic-coglycolic acid) using demineralized bone particles. Tissue Eng. Part A 14 (4), 539-547 (2008). 